\title{
On the existence of stable charged Q-balls.
}

\author{
Vieri Benci*, Donato Fortunato** \\ *Dipartimento di Matematica Applicata "U. Dini" \\ Università degli Studi di Pisa \\ Largo Bruno Pontecorvo 1/c, 56127 Pisa, Italy \\ e-mail: benci@dma.unipi.it \\ ** Dipartimento di Matematica \\ Università degli Studi di Bari Aldo Moro \\ Via Orabona 4, 70125 Bari, Italy \\ e-mail: fortunat@dm.uniba.it
}

October 9, 2018

\begin{abstract}
This paper concerns hylomorphic solitons, namely stable, solitary waves whose existence is related to the ratio energy/charge. In theoretical physics, the name $Q$-ball refers to a type of hylomorphic solitons or solitary waves relative to the Nonlinear Klein-Gordon equation (NKG). We are interested in the existence of charged Q-balls, namely Q-balls for the Nonlinear Klein-Gordon equation coupled with the Maxwell equations (NKGM). In this case the charge reduces to the electric charge. The main result of this paper establishes that stable, charged Q-balls exist provided that the interaction between matter and the gauge field is sufficiently small.
\end{abstract}

\section{Contents}

1 Introduction 2

2 Hylomorphic solitary waves and solitons 3

2.1 An abstract definition of solitary waves and solitons . . . . . . 3

2.2 Integrals of motion and hylomorphic solitons . . . . . . . . 5

3 The Nonlinear Klein-Gordon-Maxwell equations (NKGM) 8

3.1 The Klein-Gordon-Maxwell equations as Abelian gauge theory . 8

3.2 General features of the Klein-Gordon-Maxwell equations . . . . . 9

3.3 The phase space $X \ldots \ldots \ldots \ldots$ 
4 The existence result 12

4.1 Statement of the main results . . . . . . . . . . . . . . 12

4.2 Proof of the main results . . . . . . . . . . . . 13

AMS subject classification: 35C08, 35A15, 37K40, 78M30.

Key words: Q-balls, Hylomorphic solitons, Nonlinear Klein-Gordon-Maxwell equations, variational methods.

\section{Introduction}

Roughly speaking a solitary wave is a solution of a field equation whose energy travels as a localized packet and which preserves this localization in time. A soliton is a solitary wave which exhibits some form of stability so that it has a particle-like behavior (see e.g. 1], [7, [17, 20]).

Following 4, a soliton or solitary wave is called hylomorphic if its stability is due to a particular ratio between energy and the hylenic charge. The hylenic charge is an integral of motion due to a $S^{1}$-invariance of the Lagrangian (cf. section 2.2 and for more details see [4).

In theoretical physics, the name $Q$-ball refers to a type of hylomorphic solitons or solitary waves relative to the Nonlinear Klein-Gordon equation (NKG). This type of solitary waves has been first studied in the pioneering paper [18. The name Q-ball has been introduced by Coleman in $[13$. We recall that stability results for (NKG) has been established in 2, 3, 3, 11, [19. If the Klein-Gordon equations are coupled with the Maxwell equations (NKGM) then the hylenic charge reduces to the electric charge and the relative solitary waves are called charged, or gauged Q-balls (see e.g. [13]). Existence results of Q-balls for (NKGM) are stated in, 5, 6], 8, 16, however, in these papers there are not stability results and hence the existence of solitons for (NKGM) (namely stable charged Q-balls) was an open question.

The problem with the stability of charged Q-ball is that the electric charge tends to brake them since charges of the same sign repel each other. In this respect Coleman in his celebrated paper [13] says "I have been unable to construct Q-balls when the continuous symmetry is gauged. I think what is happening physically is that the long-range force caused by the gauge field forces the charge inside the Q-ball to migrate to the surface, and this destabilizes the system, but I am not sure of this".

The aim of this paper is to investigate this problem. Our main result establishes that stable charged Q-balls exist provided that the interaction between matter and gauge field is sufficiently small. Thus, this paper gives a partial answer to the problem risen by Coleman. In order to give a complete answer, we should know what happens if the interaction is strong. We conjecture that, in this case, charged stable Q-balls do not exist, but now we do not have yet a complete proof. 


\section{Hylomorphic solitary waves and solitons}

\subsection{An abstract definition of solitary waves and solitons}

Solitary waves and solitons are particular states of a dynamical system described by one or more partial differential equations. Thus, we assume that the states of this system are described by one or more fields which mathematically are represented by functions

$$
\mathbf{u}: \mathbb{R}^{N} \rightarrow V
$$

where $V$ is a vector space with norm $|\cdot|_{V}$ which is called the internal parameters space. We assume the system to be deterministic; this means that it can be described as a dynamical system $(X, \gamma)$ where $X$ is the set of the states and $\gamma: \mathbb{R} \times X \rightarrow X$ is the time evolution map. If $\mathbf{u}_{0}(x) \in X$, the evolution of the system will be described by the function

$$
\mathbf{u}(t, x):=\gamma_{t} \mathbf{u}_{0}(x)
$$

We assume that the states of $X$ have "finite energy" so that they decay at $\infty$ sufficiently fast.

We give a formal definition of solitary wave:

Definition 1 A state $\mathbf{u}(x) \in X$ is called solitary wave if

$$
\left|\gamma_{t} \mathbf{u}(x)\right|=f(x-v t)
$$

In particular, if $v=0$, then $\mathbf{u}(x)$ is called standing wave.

For example, consider a solution of a field equation having the following form

$$
\mathbf{u}(t, x)=u_{0}(x-\mathbf{v} t) e^{i(\mathbf{v} \cdot \mathbf{x}-\omega t)} ; u_{0} \in L^{2}\left(\mathbb{R}^{N}\right)
$$

then, for every $t, \mathbf{u}(t, x)$ is a solitary wave.

The solitons are solitary waves characterized by some form of stability. To define them at this level of abstractness, we need to recall some well known notions in the theory of dynamical systems.

A set $\Gamma \subset X$ is called invariant if $\forall \mathbf{u} \in \Gamma, \forall t \in \mathbb{R}, \gamma_{t} \mathbf{u} \in \Gamma$.

Definition 2 Let $(X, d)$ be a metric space and let $(X, \gamma)$ be a dynamical system. An invariant set $\Gamma \subset X$ is called stable, if $\forall \varepsilon>0, \exists \delta>0, \forall \mathbf{u} \in X$,

$$
d(\mathbf{u}, \Gamma) \leq \delta,
$$

implies that

$$
\forall t \in \mathbb{R}, d\left(\gamma_{t} \mathbf{u}, \Gamma\right) \leq \varepsilon
$$

Let $G$ be the group induced by the translations in $\mathbb{R}^{N}$, namely, for every $\tau \in \mathbb{R}^{N}$, the tranformation $g_{\tau} \in G$ is defined as follows:

$$
\left(g_{\tau} \mathbf{u}\right)(x)=\mathbf{u}(x-\tau) .
$$


A subset $\Gamma \subset X$ is called $G$-invariant if

$$
\forall \mathbf{u} \in \Gamma, \forall \tau \in \mathbb{R}^{N}, g_{\tau} \mathbf{u} \in \Gamma .
$$

Definition 3 A closed $G$-invariant set $\Gamma \subset X$ is called $G$-compact if for any sequence $\mathbf{u}_{n}(x)$ in $\Gamma$ there is a sequence $\tau_{n} \in \mathbb{R}^{N}$, such that $\mathbf{u}_{n}\left(x-\tau_{n}\right)$ has a converging subsequence.

Now we are ready to give the definition of soliton:

Definition 4 A standing wave $\mathbf{u}(x)$ is called (standing) soliton if there is an invariant set $\Gamma$ such that

- (i) $\forall t, \gamma_{t} \mathbf{u}(x) \in \Gamma$,

- (ii) $\Gamma$ is stable,

- (iii) $\Gamma$ is G-compact.

Usually, in the literature, the kind of stability described by the above definition is called orbital stability.

Remark 5 The above definition needs some explanation. For simplicity, we assume that $\Gamma$ is a manifold (actually, it is possible to prove that this is the generic case if the problem is formulated in a suitable function space). Then (iii) implies that $\Gamma$ is finite dimensional. Since $\Gamma$ is invariant, $\mathbf{u}_{0} \in \Gamma \Rightarrow \gamma_{t} \mathbf{u}_{0} \in$ $\Gamma$ for every time. Thus, since $\Gamma$ is finite dimensional, the evolution of $\mathbf{u}_{0}$ is described by a finite number of parameters. Thus the dynamical system $(\Gamma, \gamma)$ behaves as a point in a finite dimensional phase space. By the stability of $\Gamma, a$ small perturbation of $\mathbf{u}_{0}$ remains close to $\Gamma$. However, in this case, its evolution depends on an infinite number of parameters. Thus, this system appears as a finite dimensional system with a small perturbation. Since $\operatorname{dim}(G)=N$, $\operatorname{dim}(\Gamma) \geq N$ and hence, the "state" of a soliton is described by $N$ parameters which define its position and, may be, other parameters which define its "internal state".

Remark 6 We recal that (NKGM) are defined by a Lagrangian which is invariant under the action of the Lorentz group. If $\mathbf{u}_{0}$ is a stationary wave, it is possible to obtain a travelling wave just making a Lorentz boost (see e.g. [7] or [4]). More precisely, let $T_{\mathbf{v}}$ be the representation of a Lorentz boost relative to our system and let

$$
\mathbf{u}(t, x)=\gamma_{t} \mathbf{u}_{0}(x)
$$

be the evolution of our standing wave $\mathbf{u}_{0}(x)$; then

$$
\mathbf{u}^{\prime}\left(t^{\prime}, x^{\prime}\right):=T_{\mathbf{v}} \mathbf{u}(t, x)
$$

is a solution of our equation which moves in time with velocity $\mathbf{v}$. In [4] you can see the details and how this principle works in some particular cases. Obviously, if $\mathbf{u}_{0}$ is a standing soliton, $\mathbf{u}_{\mathbf{v}}$ is orbitally stable and hence it is a travelling soliton. 
Remark 7 The stability of $\Gamma \subset X$ depends on the metric of $X$; however, if $X$ is a finite dimensional vector space, all the metric (induced by a norm) are topologically equivalent; hence in this case the stability of $\Gamma \subset X$ is independent of the metric. This is not the case when $X$ is infinite dimensional. In this case the choice of the "right" metric is a delicate problem which depends on mathematical and physical considerations. In many cases, we have that

$$
\{\text { energy }\}=\{\text { positive quadratic form }\}+\{\text { higher order terms }\} .
$$

In this case, usually, it is a good choice to use the "norm of the energy":

$$
\|\cdot\|:=\sqrt{\{\text { positive quadratic form }\}}
$$

\subsection{Integrals of motion and hylomorphic solitons}

The existence and the properties of hylomorphic solitons are guaranteed by the interplay between energy $E$ and another integral of motion which, in the general case, is called hylenic charge and it will be denoted by $C$. Notice that the first integrals can be considered as functionals defined on the phase space $X$.

In this section, we shall prove some abstract theorems which guarantee the existence of hylomorphic solitons. In this paper we will be interested in the case in which $G$ is the group of translations defined by (4); however the abstract theorems hold true for any (locally compact) Lie group; similarly you do not have to assume that $E(\mathbf{u})$ and $C(\mathbf{u})$ are the energy and the hylenic charge.

Before stating the abstract theorems, we need some definitions:

Definition 8 Let $G$ be a group acting on $X$. A sequence $\mathbf{u}_{n}$ in $X$ is called $G$ compact if we can extract a subsequence $\mathbf{u}_{n_{k}}$ such that there exists a sequence $g_{k} \in G$ such that $g_{k} \mathbf{u}_{n_{k}}$ is convergent.

Definition 9 A functional $J$ on $X$ is called $G$-invariant if

$$
\forall g \in G, \forall \mathbf{u} \in X, J(g \mathbf{u})=J(\mathbf{u})
$$

Definition 10 A functional $J$ on $X$ is called $G$-compact if any minimizing sequence $\mathbf{u}_{n}$ is $G$-compact.

Remark 11 Clearly, a G-compact functional admits a minimizer. Moreover, if $J$ is $G$-invariant and $\mathbf{u}_{0}$ is a minimizers, then $\left\{g \mathbf{u}_{\mathbf{0}} \mid g \in G\right\}$ is a set of minimizer; so, if $G$ is not compact (as in the case of the translations group) and if its action is free, then the set of minimizer is not compact. This fact adds an extra difficulty to this kind of problems. $(X, \gamma)$

We make the following (abstract) assumptions on the dynamical system

- (EC-1) there are two prime integrals $E(\mathbf{u})$ and $C(\mathbf{u})$.

- (EC-2) $E(\mathbf{u})$ and $C(\mathbf{u})$ are $G$-invariant. 
Theorem 12 Assume (EC-1) and (EC-2). For $\mathbf{u} \in X$ and $e_{0}, c_{0} \in \mathbb{R}$, we set

$$
V(\mathbf{u})=\left(E(\mathbf{u})-e_{0}\right)^{2}+\left(C(\mathbf{u})-c_{0}\right)^{2} .
$$

If $V$ is $G$-compact and

$$
\Gamma=\left\{\mathbf{u} \in X: E(\mathbf{u})=e_{0}, C(\mathbf{u})=c_{0}\right\} \neq \varnothing,
$$

then every $\mathbf{u} \in \Gamma$ is a soliton.

Definition 13 A soliton which fulfills the assumptions of Th. 12 is called hylomorphic.

In order to prove Th. 12 we need the (well known) Liapunov theorem in following form:

Theorem 14 Let $\Gamma$ be an invariant set and assume that there exists a differentiable function $V$ (called a Liapunov function) such that

- (a) $V(\mathbf{u}) \geq 0$ and $V(\mathbf{u})=0 \Leftrightarrow u \in \Gamma$

- (b) $\partial_{t} V\left(\gamma_{t}(\mathbf{u})\right) \leq 0$

- (c) $V\left(\mathbf{u}_{n}\right) \rightarrow 0 \Leftrightarrow d\left(\mathbf{u}_{n}, \Gamma\right) \rightarrow 0$.

Then $\Gamma$ is stable.

Proof. For completeness, we give a proof of this well known result. Arguing by contradiction, assume that $\Gamma$, satisfying the assumptions of Th. 14, is not stable. Then there exists $\varepsilon>0$ and sequences $\mathbf{u}_{n} \in X$ and $t_{n}>0$ such that

$$
d\left(\mathbf{u}_{n}, \Gamma\right) \rightarrow 0 \text { and } d\left(\gamma_{t_{n}}\left(\mathbf{u}_{n}\right), \Gamma\right)>\varepsilon .
$$

Then we have

$$
d\left(\mathbf{u}_{n}, \Gamma\right) \rightarrow 0 \Longrightarrow V\left(\mathbf{u}_{n}\right) \rightarrow 0 \Longrightarrow V\left(\gamma_{t_{n}}\left(\mathbf{u}_{n}\right)\right) \rightarrow 0 \Longrightarrow d\left(\gamma_{t_{n}}\left(\mathbf{u}_{n}\right), \Gamma\right) \rightarrow 0
$$

where the first and the third implications are consequence of property (c). The second implication follows from property (b). Clearly, this fact contradicts (8).

Now we are ready to prove Theorem $\mathbf{1 2}$

Proof of Th. 12 We have to prove that $\Gamma$ in (7) satisfies (i),(ii) and (iii) of Def. (4). The property (iii), namely the fact that $\Gamma$ is G-compact, is a trivial consequence of the fact that $\Gamma$ is the set of minimizers of a G-compact functional $V$. The invariance property (i) is clearly satisfied since $E$ and $C$ are constants of the motion. It remains to prove (ii), namely that $\Gamma$ is stable. To this end we shall use Th. 14. So we need to show that $V(\mathbf{u})$ satisfies (a), (b) and (c). Statements (a) and (b) are trivial. Now we prove (c). First we show the 
implication $\Rightarrow$. Let $\mathbf{u}_{n}$ be a sequence such that $V\left(\mathbf{u}_{n}\right) \rightarrow 0$. By contradiction we assume that $d\left(\mathbf{u}_{n}, \Gamma\right) \nrightarrow 0$, namely that there is a subsequence $\mathbf{u}_{n}^{\prime}$ such that

$$
d\left(\mathbf{u}_{n}^{\prime}, \Gamma\right) \geq a>0 .
$$

Since $V\left(\mathbf{u}_{n}\right) \rightarrow 0$ also $V\left(\mathbf{u}_{n}^{\prime}\right) \rightarrow 0$, and, since $V$ is $G$ compact, there exists a sequence $g_{n}$ in $G$ such that, for a subsequence $\mathbf{u}_{n}^{\prime \prime}$, we have $g_{n} \mathbf{u}_{n}^{\prime \prime} \rightarrow \mathbf{u}_{0}$. Then

$$
d\left(\mathbf{u}_{n}^{\prime \prime}, \Gamma\right)=d\left(g_{n} \mathbf{u}_{n}^{\prime \prime}, \Gamma\right) \leq d\left(g_{n} \mathbf{u}_{n}^{\prime \prime}, \mathbf{u}_{0}\right) \rightarrow 0
$$

and this contradicts (9).

Now we prove the other implication $\Leftarrow$. Let $\mathbf{u}_{n}$ be a sequence such that $d\left(\mathbf{u}_{n}, \Gamma\right) \rightarrow 0$, then there exists $\mathbf{v}_{n} \in \Gamma$ s.t.

$$
d\left(\mathbf{u}_{n}, \Gamma\right) \geq d\left(\mathbf{u}_{n}, \mathbf{v}_{n}\right)-\frac{1}{n} .
$$

Since $V$ is G-compact, also $\Gamma$ is G-compact; so, for a suitable sequence $g_{n}$, we have $g_{n} \mathbf{v}_{n} \rightarrow \overline{\mathbf{w}} \in \Gamma$. We get the conclusion if we show that $V\left(\mathbf{u}_{n}\right) \rightarrow 0$. We have by (10), that $d\left(\mathbf{u}_{n}, \mathbf{v}_{n}\right) \rightarrow 0$ and hence $d\left(g_{n} \mathbf{u}_{n}, g_{n} \mathbf{v}_{n}\right) \rightarrow 0$ and so, since $g_{n} \mathbf{v}_{n} \rightarrow \overline{\mathbf{w}}$, we have $g_{n} \mathbf{u}_{n} \rightarrow \overline{\mathbf{w}} \in \Gamma$. Therefore, by the continuity of $V$ and since $\overline{\mathbf{w}} \in \Gamma$, we have $V\left(g_{n} \mathbf{u}_{n}\right) \rightarrow V(\overline{\mathbf{w}})=0$ and we can conclude that $V\left(\mathbf{u}_{n}\right) \rightarrow 0$.

In the cases in which we are interested, $X$ is an infinite dimensional manifold; then if you choose generic $e_{0}$ and $c_{0}, V$ is not $G$-compact since the set $\Gamma=$ $\left\{\mathbf{u} \in X: E(\mathbf{u})=e_{0}, C(\mathbf{u})=c_{0}\right\}$ has codimension 2 .

The following theorem will be useful to determine $e_{0}$ and $c_{0}$ in such a way that $V$ is $G$-compact and hence to prove the existence of solitons by using Theorem 12. It is based on a penalization of the so called hylomorphy ratio $\frac{E(\mathbf{u})}{C(\mathbf{u})}$.

Theorem 15 Assume that the dynamical system $(X, \gamma)$ satisfies (EC-1) and (EC-2). Moreover we set

$$
J(\mathbf{u})=\frac{E(\mathbf{u})}{|C(\mathbf{u})|}+\delta E(\mathbf{u})^{2}
$$

where $\delta$ is a positive constant and assume that $J$ is $G$-compact. Then $J(\mathbf{u})$ has a minimizer $\mathbf{u}_{0}$. Moreover, if we set $e_{0}=E\left(\mathbf{u}_{0}\right), c_{0}=C\left(\mathbf{u}_{0}\right)$, any $\mathbf{u} \in X$ such that $E(\mathbf{u})=e_{0}, C(\mathbf{u})=c_{0}$, is an (hylomorphic) soliton for $(X, \gamma)$.

Proof. Let $\mathbf{u}_{n}$ be a minimizing sequence of $J . J$ is $G$-compact, then,for a suitable subsequence $\mathbf{u}_{n_{k}}$ and a suitable sequence $g_{k}$, we get $g_{k} \mathbf{u}_{n_{k}} \rightarrow \mathbf{u}_{0}$. Clearly $\mathbf{u}_{0}$ is a minimizer of $J$. Now set $e_{0}=E\left(\mathbf{u}_{0}\right), c_{0}=\left|C\left(\mathbf{u}_{0}\right)\right|$ and

$$
V(\mathbf{u})=\left(E(\mathbf{u})-e_{0}\right)^{2}+\left(C(\mathbf{u})-c_{0}\right)^{2} .
$$


We show that $V$ is $G$-compact: let $\mathbf{w}_{n}$ be a minimizing sequence for $V$, then $V\left(\mathbf{w}_{n}\right) \rightarrow 0$ and consequently $E\left(\mathbf{w}_{n}\right) \rightarrow e_{0}$ and $C\left(\mathbf{w}_{n}\right) \rightarrow c_{0}$. Now, since

$$
\min J=J\left(\mathbf{u}_{0}\right)=\frac{e_{0}}{c_{0}}+\delta e_{0}^{2},
$$

we have that $\mathbf{w}_{n}$ is a minimizing minimizing sequence also for $J$. Then, since $J$ is $G$-compact, we get

$$
\mathbf{w}_{n} \text { is } G \text {-compact. }
$$

So we conclude that $V$ is $G$-compact and hence the conclusion follows by using Theorem 12

Remark 16 The reason why $J(\mathbf{u})$ has such an awkward form depends on the fact that in our concrete applications is just this form which guarantees the $G$-compactness.

\section{The Nonlinear Klein-Gordon-Maxwell equa- tions (NKGM)}

\subsection{The Klein-Gordon-Maxwell equations as Abelian gauge theory}

The nonlinear Klein-Gordon equation for a complex valued field $\psi$, defined on the spacetime $\mathbb{R}^{4}$, can be written as follows:

$$
\square \psi+W^{\prime}(\psi)=0
$$

where

$$
\square \psi=\frac{\partial^{2} \psi}{\partial t^{2}}-\Delta \psi, \quad \Delta \psi=\frac{\partial^{2} \psi}{\partial x_{1}^{2}}+\frac{\partial^{2} \psi}{\partial x_{2}^{2}}+\frac{\partial^{2} \psi}{\partial x_{3}^{2}}
$$

and, with some abuse of notation,

$$
W^{\prime}(\psi)=F^{\prime}(|\psi|) \frac{\psi}{|\psi|}
$$

for some smooth function $F:[0, \infty) \rightarrow \mathbb{R}$. Hereafter $x=\left(x_{1}, x_{2}, x_{3}\right)$ and $t$ will denote the space and time variables. The field $\psi: \mathbb{R}^{4} \rightarrow \mathbb{C}$ will be called matter field. If $W^{\prime}(s)$ is linear, $W^{\prime}(s)=m^{2} s, m \neq 0$, equation (13) reduces to the Klein-Gordon equation. We assume that

$$
W(s)=\frac{m^{2}}{2} s^{2}+N(s), \quad m>0, N(s)=o\left(s^{2}\right) .
$$

Consider the Abelian gauge theory in $\mathbb{R}^{4}$ equipped with the Minkowski metric and described by the Lagrangian density (see e.g. [7, 21])

$$
\mathcal{L}=\mathcal{L}_{0}+\mathcal{L}_{1}-W(|\psi|)
$$


where

$$
\begin{gathered}
\mathcal{L}_{0}=\frac{1}{2}\left(\left|D_{\varphi} \psi\right|^{2}-\left|D_{\mathbf{A}} \psi\right|^{2}\right) \\
\mathcal{L}_{1}=\frac{1}{2}\left(\left|\partial_{t} \mathbf{A}+\nabla \phi\right|^{2}-|\nabla \times \mathbf{A}|^{2}\right) .
\end{gathered}
$$

Here $q$ denotes a positive parameter which, in some physical model, represents the unit electric charge, $\nabla \times$ and $\nabla$ denote respectively the curl and the gradient operators;

$$
\mathbf{A}=\left(A_{1}, A_{2}, A_{3}\right) \in \mathbb{R}^{3} \text { and } \phi \in \mathbb{R}
$$

are the gauge potentials;

$$
D_{\varphi} \psi=\left(\partial_{t}+i q \phi\right) \psi
$$

is the covariant derivatives with respect to the $t$ variable, and

$$
D_{\mathbf{A}} \psi=(\nabla-i q \mathbf{A}) \psi
$$

is the covariant derivatives with respect to the $x$ variable (see for example [7] and 21]).

Now consider the total action

$$
\mathcal{S}=\int\left(\mathcal{L}_{0}+\mathcal{L}_{1}-W(|\psi|)\right) d x d t .
$$

Making the variation of $\mathcal{S}$ with respect to $\psi, \phi$ and $\mathbf{A}$, we get the system of the so called Nonlinear-Klein-Gordon-Maxwell equations (NKGM):

$$
\begin{gathered}
D_{\varphi}^{2} \psi-D_{\mathbf{A}}^{2} \psi+W^{\prime}(\psi)=0 \\
\nabla \cdot\left(\partial_{t} \mathbf{A}+\nabla \phi\right)=q \operatorname{Re}\left(i D_{\varphi} \psi \bar{\psi}\right) \\
\nabla \times(\nabla \times \mathbf{A})+\partial_{t}\left(\partial_{t} \mathbf{A}+\nabla \phi\right)=q \operatorname{Re}\left(i D_{\mathbf{A}} \psi \bar{\psi}\right) .
\end{gathered}
$$

Here $\nabla \cdot$ denotes the divergence operator.

\subsection{General features of the Klein-Gordon-Maxwell equa- tions}

If we make the following change of variables:

$$
\begin{gathered}
\mathbf{E}=-\left(\frac{\partial \mathbf{A}}{\partial t}+\nabla \phi\right) \\
\mathbf{H}=\nabla \times \mathbf{A} \\
\rho=-q \operatorname{Re}\left(i D_{\varphi} \psi \bar{\psi}\right) \\
\mathbf{j}=q \operatorname{Re}\left(i D_{\mathbf{A}} \psi \bar{\psi}\right),
\end{gathered}
$$

we see that (18) and (19) are the second couple of the Maxwell equations (Gauss and Ampere laws) with respect to a matter distribution whose electric charge 
and current densities are respectively $\rho$ and $\mathbf{j}$. These equations can be written as follows:

$$
\begin{gathered}
\nabla \cdot \mathbf{E}=\rho \\
\nabla \times \mathbf{H}-\frac{\partial \mathbf{E}}{\partial t}=\mathbf{j} .
\end{gathered}
$$

Equations (20) and (21) give rise to the first couple of the Maxwell equations:

$$
\begin{gathered}
\nabla \times \mathbf{E}+\frac{\partial \mathbf{H}}{\partial t}=0 \\
\nabla \cdot \mathbf{H}=0 .
\end{gathered}
$$

Sometimes it is useful to give a different form to these equations; if we write $\psi$ in polar form

$$
\psi(x, t)=u(x, t) e^{i S(x, t)}, \quad u \geq 0, \quad S \in \mathbb{R} / 2 \pi \mathbb{Z}
$$

equation (17) can be split in the two following ones

$$
\begin{gathered}
\square u+W^{\prime}(u)+\left[|\nabla S-q \mathbf{A}|^{2}-\left(\frac{\partial S}{\partial t}+q \phi\right)^{2}\right] u=0 \\
\frac{\partial}{\partial t}\left[\left(\frac{\partial S}{\partial t}+q \phi\right) u^{2}\right]-\nabla \cdot\left[(\nabla S-q \mathbf{A}) u^{2}\right]=0 .
\end{gathered}
$$

Observe that, using the polar form (24), (22) and (23) become

$$
\rho=-q\left(\frac{\partial S}{\partial t}+q \phi\right) u^{2}, \mathbf{j}=q(\nabla S-q \mathbf{A}) u^{2} .
$$

Then equations (25) and (26), using the variables $\mathbf{j}$ and $\rho$, can be written as follows:

$$
\begin{gathered}
\square u+W^{\prime}(u)+\frac{\mathbf{j}^{2}-\rho^{2}}{q^{2} u^{3}}=0 \\
\frac{\partial \rho}{\partial t}+\nabla \cdot \mathbf{j}=0 .
\end{gathered}
$$

Equation (27) is the charge continuity equation.

Notice that equation (27) is also a consequence of (GAUSS) and (AMPERE) and hence it can be eliminated. Thus equations (17/18/19) are equivalent to equations (GAUSS, AMPERE FARADAY, NOMONOPOLE MATTER).

In conclusion, an Abelian gauge theory, via equations (GAUSS, AMPERE FARADAY, NOMONOPOLE MATTER), provides a model of interaction of the matter field $\psi$ with the electromagnetic field $(\mathbf{E}, \mathbf{H})$.

Observe that the Lagrangian (15) is invariant with respect to the gauge transformations

$$
\begin{gathered}
\psi \rightarrow e^{i q \chi} \psi \\
\phi \rightarrow \phi-\partial_{t} \chi
\end{gathered}
$$




$$
\mathbf{A} \rightarrow \mathbf{A}+\nabla \chi
$$

where $\chi \in C^{\infty}\left(\mathbb{R}^{4}\right)$.

So, our equations are gauge invariant; if we use the variables $u, \rho, \mathbf{j}, \mathbf{E}, \mathbf{H}$, this fact can be checked directly since these variables are gauge invariant. Actually, equations (GAUSS, AMPERE FARADAY, NOMONOPOLE, MATTER) are the gauge invariant formulation of equations (17/18/19).

\subsection{The phase space $X$}

Noether's theorem states that any invariance for a one-parameter group of the Lagrangian implies the existence of an integral of motion (see e.g. [14, 44). In particular the invariants, which are relevant for us, are the energy and the charge, which, in the gauge invariant variables, take the following form (for the explicit computation of $E$ see e.g. 7])

$$
\begin{gathered}
E(\mathbf{u})=\frac{1}{2} \int\left(\left|\partial_{t} u\right|^{2}+|\nabla u|^{2}+\frac{\rho^{2}+\mathbf{j}^{2}}{q^{2} u^{2}}+\mathbf{E}^{2}+\mathbf{H}^{2}\right) d x+\int W(u) d x \\
C_{e l}=\int \rho d x .
\end{gathered}
$$

Observe that $C_{e l}$ is the electric charge and $C=\frac{C_{e l}}{q}$ is the hylenic charge (as defined e.g. in [4]).

The term $\left(\rho^{2}+\mathbf{j}^{2}\right) / u^{2}$ is singular and the energy does not have the form (57). In order to avoid this problem, it is convenient to introduce new gauge invariant variables which eliminate this singularity:

$$
\theta=\frac{-\rho}{q u} ; \Theta=\frac{\mathbf{j}}{q u}
$$

Using these new variables the energy takes the form:

$$
\begin{aligned}
E(\mathbf{u}) & =\frac{1}{2} \int\left(\left|\partial_{t} u\right|^{2}+|\nabla u|^{2}+\theta^{2}+\Theta^{2}+\mathbf{E}^{2}+\mathbf{H}^{2}\right) d x+\int W(u) d x \\
& =\frac{1}{2} \int\left[\left|\partial_{t} u\right|^{2}+|\nabla u|^{2}+m^{2} u^{2}+\theta^{2}+\Theta^{2}+\mathbf{E}^{2}+\mathbf{H}^{2}\right]+\int N(u) .
\end{aligned}
$$

Thus, we can construct the space $X$ and its metric taking into account the suggestions of remark 7 The generic point in the phase space is given by

$$
\mathbf{u}=(u, \hat{u}, \theta, \Theta, \mathbf{E}, \mathbf{H})
$$

where $\hat{u}=\partial_{t} u$ is considered as independent variable; the phase space is given by

$$
X=\{\mathbf{u} \in \mathcal{H}: \nabla \cdot \mathbf{E}=-q \theta u, \nabla \cdot \mathbf{H}=0\}
$$

where $\mathcal{H}$ is the Hilbert space of the functions

$$
\mathbf{u}=(u, \hat{u}, \theta, \Theta, \mathbf{E}, \mathbf{H}) \in H^{1}\left(\mathbb{R}^{3}\right) \times L^{2}\left(\mathbb{R}^{3}\right)^{11}
$$


equipped with the norm defined by the quadratic part of the energy:

$$
\|\mathbf{u}\|^{2}=\int\left[\hat{u}^{2}+|\nabla u|^{2}+m^{2} u^{2}+\theta^{2}+\Theta^{2}+\mathbf{E}^{2}+\mathbf{H}^{2}\right] d x
$$

In these new variables the energy and the hylenic charge become two continuous functionals on $X$ having the form

$$
\begin{gathered}
E(\mathbf{u})=\frac{1}{2} \int\left(\left|\hat{u}^{2}\right|^{2}+|\nabla u|^{2}+\theta^{2}+\Theta^{2}+\mathbf{E}^{2}+\mathbf{H}^{2}\right) d x+\int W(u) d x \\
C(\mathbf{u})=\int \theta u d x .
\end{gathered}
$$

Our equations (MATTER, GAUSS, AMPERE, FARADAY, NOMONOPOLE) become

$$
\begin{aligned}
\square u+W^{\prime}(u)+\frac{\Theta^{2}-\theta^{2}}{u} & =0 \\
\nabla \cdot \mathbf{E} & =-q \theta u \\
\nabla \times \mathbf{H}-\frac{\partial \mathbf{E}}{\partial t} & =q \Theta u \\
\nabla \times \mathbf{E}+\frac{\partial \mathbf{H}}{\partial t} & =0 \\
\nabla \cdot \mathbf{H} & =0 .
\end{aligned}
$$

Remark 17 In the following we shall assume that the Cauchy problem for (NKGM) is well posed in X. Actually, in the literature there are few results relative to this problem (we know only [15]) and we do not know which are the assumptions that $W$ should satisfy. Also, we refer to [10] for a discussion and some partial result on this issue.

\section{The existence result}

\subsection{Statement of the main results}

We make the following assumptions:

- (W-i) (Positivity) $W(s) \geq 0$

- (W-ii) (Nondegeneracy) $W=W(s)(s \geq 0)$ is $C^{2}$ near the origin with $W(0)=W^{\prime}(0)=0 ; W^{\prime \prime}(0)=m^{2}>0$

- (W-iii) (Hylomorphy) $\exists \bar{s}>0$ and $\alpha \in(0, m)$ such that $W(\bar{s}) \leq \frac{1}{2} \alpha^{2} \bar{s}^{2}$

- (W-iiii)(Growth condition) There are constants $a, b>0,6>p>2$ s.t. $\left|N^{\prime}(s)\right| \leq a s^{p-1}+b s^{2-\frac{2}{p}}$ where $N$ id defined by eq. (14). 
Here there are some comments on assumptions (W-i), (W-ii), (W-iii), (Wiiii).

(W-i) Clearly (see (33)) (W-i) implies that the energy is positive; if this condition does not hold, it is possible to have solitary waves, but not hylomorphic waves (cf. the discussion in section 4.2 of [6]).

(W-ii) In order to have solitary waves it is necessary to have $W^{\prime \prime}(0) \geq 0$. There are some results also when $W^{\prime \prime}(0)=0$ (null-mass case, see e.g. [12]), however the most interesting situation occurs when $W^{\prime \prime}(0)>0$.

(W-iii) This is the crucial assumption which characterizes the potentials which might produce hylomorphic solitons. As we will see, this assumption permits to have states $\mathbf{u}$ with hylomorphy ratio $\frac{E(\mathbf{u})}{C(\mathbf{u})}<m$. By this assumption there exists $s_{0}$ such that $N\left(s_{0}\right)<0$

(W-iiii) This assumption contains the usual growth condition at infinity which guarantees the $C^{1}$ regularity of the functional. Moreover it implies that $\left|N^{\prime}(s)\right|=O\left(s^{2-\frac{2}{p}}\right)$ for $s$ small.

We have the following results:

Theorem 18 Assume that (W-i),(W-ii),(W-iii),(W-iiii) hold, then there exists $\bar{q}$ such that for every $q \in[0, \bar{q}]$, equations (35) have a continuous family $\mathbf{u}_{\delta}$ $(\delta \in(0, \bar{\delta}(q)))$ of independent, hylomorphic solitons (two solitons $\mathbf{u}_{\delta_{1}}, \mathbf{u}_{\delta_{2}}$ are called independent if $\mathbf{u}_{\delta_{1}} \neq g \mathbf{u}_{\delta_{2}}$ for every $g \in G$ ).

Theorem 19 The solitons $\mathbf{u}_{\delta}=\left(u_{\delta}, \hat{u}_{\delta}, \theta_{\delta}, \Theta_{\delta}, \mathbf{E}_{\delta}, \mathbf{H}_{\delta}\right)$ in Theorem 18 are stationary solutions of (35), this means that $\hat{u}_{\delta}=\Theta_{\delta}=\mathbf{H}_{\delta}=0, \mathbf{E}_{\delta}=-\nabla \phi_{\delta}$ and $u_{\delta}, \theta_{\delta}, \phi_{\delta}$ solve the equations

$$
\begin{aligned}
-\Delta u_{\delta}+W^{\prime}\left(u_{\delta}\right)-\frac{\theta_{\delta}^{2}}{u_{\delta}} & =0 \\
-\Delta \phi_{\delta} & =-q \theta_{\delta} u_{\delta}
\end{aligned}
$$

The proofs of Theorem 18 and of Theorem 19 will be given in the next section.

\subsection{Proof of the main results}

First of all we introduce the following notation:

$$
\begin{gathered}
Q=\left\{x=\left(x_{1}, \ldots, x_{N}\right) \in \mathbb{R}^{N}: 0 \leq x_{i}<1, i=1, . ., N\right\} \\
Q_{j}=j+Q, j \in \mathbb{Z}^{3} \\
X\left(Q_{j}\right)=\left\{\left.\mathbf{u}\right|_{Q_{j}}: \mathbf{u} \in X\right\} \\
\|\mathbf{u}\|_{Q_{j}}^{2}=\int_{Q_{j}}\left[\hat{u}^{2}+|\nabla u|^{2}+m^{2} u^{2}+\theta^{2}+\Theta^{2}+\mathbf{E}^{2}+\mathbf{H}^{2}\right] d x
\end{gathered}
$$




$$
\begin{gathered}
E_{Q_{j}}(\mathbf{u})=\frac{1}{2} \int_{Q_{j}}\left[|\hat{u}|^{2}+\theta^{2}+|\nabla u|^{2}+\Theta^{2}+u^{2}+\mathbf{E}^{2}+\mathbf{H}^{2}\right] d x+\int_{Q_{j}} W(u) d x \\
C_{Q_{j}}(\mathbf{u})=\int_{Q_{j}} \theta u d x
\end{gathered}
$$

and

$$
\Lambda_{0}=\inf _{\mathbf{u} \in X} \frac{\frac{1}{2}\|\mathbf{u}\|_{Q}^{2}}{\left|C_{Q}(\mathbf{u})\right|}, \Lambda_{*}=\inf _{\mathbf{u} \in X} \frac{E(\mathbf{u})}{|C(\mathbf{u})|}=\inf _{\mathbf{u} \in X} \frac{\frac{1}{2}\|\mathbf{u}\|^{2}+\int N(u) d x}{|C(\mathbf{u})|}
$$

Lemma 20 The following inequality holds:

$$
\Lambda_{0} \geq m
$$

Proof:

$$
\begin{aligned}
\Lambda_{0} & =\inf _{\mathbf{u} \in X} \frac{\frac{1}{2}\|\mathbf{u}\|_{Q}^{2}}{\left|C_{Q}(\mathbf{u})\right|} \\
& \geq \inf _{\mathbf{u} \in X} \frac{\frac{1}{2} \int_{Q}\left[\hat{u}^{2}+|\nabla u|^{2}+m^{2} u^{2}+\theta^{2}+\Theta^{2}+\mathbf{E}^{2}+\mathbf{H}^{2}\right] d x}{\int_{Q}|\theta u| d x} \\
& \geq \inf _{\mathbf{u} \in X} \frac{\frac{1}{2} \int_{Q}\left[m^{2} u^{2}+\theta^{2}\right] d x}{\int_{Q}|\theta u| d x}=\inf _{\mathbf{u} \in X} \frac{\int_{Q} m|u||\theta| d x}{\int_{Q}|\theta u| d x} \geq m
\end{aligned}
$$

The next lemma provides a crucial estimate for the existence of solitons:

Lemma 21 Let $\alpha, \bar{s}, m$ be the positive constants appearing in (W-iii). Then there is a positive constant $c$ such that for any $0<q<\frac{c}{s} \sqrt{(m-\alpha)^{3} \alpha}$ we have

$$
\Lambda_{*}<m
$$

Proof: We set

$$
u_{R}=\left\{\begin{array}{cc}
\bar{s} & \text { if }|x|<R \\
0 & \text { if }|x|>R+1 \\
\frac{|x|}{R} \bar{s}-(|x|-R) \frac{R+1}{R} \bar{s} & \text { if } R<|x|<R+1
\end{array}\right.
$$

where $R>1$. Moreover we denote by $\varphi_{R} \in \mathcal{D}^{1,2}$ the solution of the following equation

$$
\Delta \varphi=-q \alpha u_{R}^{2}
$$


We have

$$
\begin{aligned}
\Lambda_{*} & =\inf _{\mathbf{u} \in X} \frac{\frac{1}{2}\|\mathbf{u}\|^{2}+\int N(u) d x}{|C(\mathbf{u})|} \\
& =\inf _{\mathbf{u} \in X} \frac{\frac{1}{2} \int\left[\hat{u}^{2}+|\nabla u|^{2}+\theta^{2}+\Theta^{2}+\mathbf{E}^{2}+\mathbf{H}^{2}\right] d x+\int W(u) d x}{\left|\int \theta u d x\right|} .
\end{aligned}
$$

Now remember that $\mathbf{u}=(u, \hat{u}, \theta, \Theta, \mathbf{E}, \mathbf{H})$ and take $\mathbf{u}=\mathbf{u}_{R}$ with

$$
\mathbf{u}_{R}=\left(u_{R}, 0, \alpha u_{R}, 0, \nabla \varphi_{R}, \mathbf{0}\right) .
$$

By (31) and (40), $\mathbf{u}_{R} \in X$; then we have

$$
\begin{aligned}
\Lambda_{*} & =\inf _{\mathbf{u} \in X} \frac{\frac{1}{2}\|\mathbf{u}\|^{2}+\int N(u) d x}{|C(\mathbf{u})|} \leq \frac{\frac{1}{2}\left\|\mathbf{u}_{R}\right\|^{2}+\int N\left(u_{R}\right) d x}{C\left(\mathbf{u}_{R}\right)} \\
& =\frac{\frac{1}{2} \int\left[\left|\nabla u_{R}\right|^{2}+\alpha^{2} u_{R}^{2}+\left|\nabla \varphi_{R}\right|^{2}\right] d x+\int W\left(u_{R}\right) d x}{\alpha \int u_{R}^{2} d x} \\
& \leq \frac{\frac{1}{2} \int_{|x|<R}\left[\left|\nabla u_{R}\right|^{2}+\alpha^{2} u_{R}^{2}\right]+\int_{|x|<R} W\left(u_{R}\right)}{\alpha \int_{|x|<R} u_{R}^{2} d x} \\
& +\frac{\frac{1}{2} \int_{R<|x|<R+1}\left[\left|\nabla u_{R}\right|^{2}+\alpha^{2} u_{R}^{2}\right]+\int_{R<|x|<R+1} W\left(u_{R}\right)}{\alpha \int_{|x|<R} u_{R}^{2}}+\frac{\frac{1}{2} \int\left|\nabla \varphi_{R}\right|^{2}}{\alpha \int_{|x|<R} u_{R}^{2}} \\
& =\frac{\frac{1}{2} \int_{|x|<R} \alpha^{2} \bar{s}^{2}+\int_{|x|<R} W(\bar{s})}{\alpha \int_{|x|<R} \bar{s}^{2}}+\frac{c_{1} R^{2}}{\alpha \int_{|x|<R} \bar{s}^{2}}+\frac{\frac{1}{2} \int\left|\nabla \varphi_{R}\right|^{2}}{\alpha \int_{|x|<R} \bar{s}^{2}} \\
& \leq \alpha+\frac{c_{2}}{\alpha R}+\frac{\frac{1}{2} \int\left|\nabla \varphi_{R}\right|^{2}}{\frac{4}{3} \pi \alpha \bar{s}^{2} R^{3}}
\end{aligned}
$$

where the last inequality is a consequence of (W-iii).

In order to estimate the term containing $\varphi_{R}$ in (41), we remember that $\varphi_{R}$ is the solution of (40). Observe that $u_{R}^{2}$ has radial symmetry and that the electric field outside any spherically symmetric charge distribution is the same as if all of the charge were concentrated into a point. So $\left|\nabla \varphi_{R}(r)\right|$ corresponds to the strength of an electrostatic field at distance $r$, created by an electric charge given by

$$
\left|C_{e l}\right|=\int_{|x| \leq r} q \alpha u_{R}^{2} d x=4 \pi \int_{0}^{r} q \alpha u_{R}^{2} v^{2} d v
$$

and located at the origin. So we have

$$
\left|\nabla \varphi_{R}(r)\right|=\frac{\left|C_{e l}\right|}{r^{2}}\left\{\begin{array}{cc}
=\frac{4}{3} \pi q \alpha \bar{s}^{2} r & \text { if } r<R \\
\leq \frac{4}{3} \pi q \alpha \bar{s}^{2} \frac{(R+1)^{3}}{r^{2}} & \text { if } r \geq R
\end{array}\right.
$$


Then

$$
\begin{aligned}
\int\left|\nabla \varphi_{R}\right|^{2} d x & \leq c_{3} q^{2} \alpha^{2} \bar{s}^{4}\left(\int_{r<R} r^{4} d r+\int_{r>R} \frac{(R+1)^{6}}{r^{2}} d r\right) \\
& \leq c_{4} q^{2} \alpha^{2} \bar{s}^{4}\left(R^{5}+\frac{(R+1)^{6}}{R}\right) \leq c_{5} q^{2} \alpha^{2} \bar{s}^{4} R^{5}
\end{aligned}
$$

Then

$$
\frac{\frac{1}{2} \int\left|\nabla \varphi_{R}\right|^{2}}{\frac{4}{3} \pi \alpha \bar{s}^{2} R^{3}} \leq c_{6} q^{2} \alpha \bar{s}^{2} R^{2} .
$$

By (41) and (42), we get

$$
\Lambda_{*} \leq \alpha+\frac{c_{1}}{\alpha R}+c_{6} q^{2} \alpha \bar{s}^{2} R^{2} .
$$

Now set

$$
m-\alpha=2 \varepsilon
$$

and take

$$
R=\frac{c_{1}}{\alpha \varepsilon}, 0<q<\sqrt{\frac{\varepsilon^{3} \alpha}{\bar{s}^{2} c_{1}^{2} c_{6}}} .
$$

With these choices of $R$ and $q$, a direct calculation shows that

$$
\alpha+\frac{c_{1}}{\alpha R}+c_{6} q^{2} \alpha \bar{s}^{2} R^{2}<m .
$$

Then, by (43) and (44), we get that there exists a positive constant $c$ such that, for $0<q<\frac{c}{s} \sqrt{(m-\alpha)^{3} \alpha}$, we have

$$
\Lambda_{*}<m .
$$

Lemma 22 Consider any sequence

$$
\mathbf{u}_{n}=\mathbf{u}+\mathbf{w}_{n} \in X
$$

where $\mathbf{w}_{n}$ converges weakly to 0 . Then

$$
E\left(\mathbf{u}_{n}\right)=E(\mathbf{u})+E\left(\mathbf{w}_{n}\right)+o(1)
$$

and

$$
C\left(\mathbf{u}_{n}\right)=C(\mathbf{u})+C\left(\mathbf{w}_{n}\right)+o(1) .
$$


Proof. First of all we introduce the following notation:

As usual $u, w_{n}$ will denote the first components respectively of $\mathbf{u}, \mathbf{w}_{n} \in$ $H^{1}\left(\mathbb{R}^{3}\right) \times L^{2}\left(\mathbb{R}^{3}\right)^{11}$.

If $v \in H^{1}\left(\mathbb{R}^{3}\right)$, we set

$$
K(v)=\int N(v) d x
$$

and for any measurable $A \subset \mathbb{R}^{N}$,

$$
K_{A}(v)=\int_{A} N(v) d x
$$

We have to show that $\lim _{n \rightarrow \infty}\left|E\left(\mathbf{u}+\mathbf{w}_{n}\right)-E(\mathbf{u})-E\left(\mathbf{w}_{n}\right)\right|=0$. By (32), (33) we have that

$$
\begin{aligned}
& \lim _{n \rightarrow \infty}\left|E\left(\mathbf{u}+\mathbf{w}_{n}\right)-E(\mathbf{u})-E\left(\mathbf{w}_{n}\right)\right| \\
& \leq \lim _{n \rightarrow \infty} \frac{1}{2}\left|\left\|\mathbf{u}+\mathbf{w}_{n}\right\|^{2}-\|\mathbf{u}\|^{2}-\left\|\mathbf{w}_{n}\right\|^{2}\right|+\lim _{n \rightarrow \infty}\left|K\left(u+w_{n}\right)-K(u)-K\left(w_{n}\right)\right| .
\end{aligned}
$$

Let us consider each piece independently. If $(\cdot, \cdot)$ denotes the inner product induced by the norm \|\| we have:

$$
\lim _{n \rightarrow \infty}\left|\left\|\mathbf{u}+\mathbf{w}_{n}\right\|^{2}-\|\mathbf{u}\|^{2}-\left\|\mathbf{w}_{n}\right\|^{2}\right|=\lim _{n \rightarrow \infty}\left|2\left(\mathbf{u}, \mathbf{w}_{n}\right)\right|=0 .
$$

Choose $\varepsilon>0$ and $R=R(\varepsilon)>0$ such that

$$
\left|K_{B_{R}^{c}}(u)\right|<\varepsilon
$$

where

$$
B_{R}^{c}=\mathbb{R}^{N}-B_{R} \text { and } B_{R}=\left\{x \in \mathbb{R}^{N}:|x|<R\right\} .
$$

Since $w_{n} \rightarrow 0$ weakly in $H^{1}\left(\mathbb{R}^{3}\right)$, by usual compactness arguments, we have that

$$
K_{B_{R}}\left(w_{n}\right) \rightarrow 0 \text { and } K_{B_{R}}\left(u+w_{n}\right) \rightarrow K_{B_{R}}(u) .
$$

Then, by (48) and (49), we have

$$
\begin{aligned}
& \lim _{n \rightarrow \infty}\left|K\left(u+w_{n}\right)-K(u)-K\left(w_{n}\right)\right| \\
& =\lim _{n \rightarrow \infty}\left|K_{B_{R}^{c}}\left(u+w_{n}\right)+K_{B_{R}}\left(u+w_{n}\right)-K_{B_{R}^{c}}(u)-K_{B_{R}}(u)-K_{B_{R}^{c}}\left(w_{n}\right)-K_{B_{R}}\left(w_{n}\right)\right| \\
& =\lim _{n \rightarrow \infty}\left|K_{B_{R}^{c}}\left(u+w_{n}\right)-K_{B_{R}^{c}}(u)-K_{B_{R}^{c}}\left(w_{n}\right)\right| \\
& \leq \lim _{n \rightarrow \infty}\left|K_{B_{R}^{c}}\left(u+w_{n}\right)-K_{B_{R}^{c}}\left(w_{n}\right)\right|+\varepsilon .
\end{aligned}
$$

Now observe that, for $\Omega \subset \mathbb{R}^{3}$, we have that

$$
K^{\prime} \text { is bounded from } H^{1}(\Omega) \text { into its dual }\left(H^{1}(\Omega)\right)^{\prime}
$$


In fact let $v_{n}$ be a bounded sequence in $H^{1}(\Omega)$, then, by the growth assumption (W-iii) we have

$$
\left|N^{\prime}\left(v_{n}\right)\right| \leq a\left|v_{n}\right|^{p-1}+b\left|v_{n}\right|^{2-\frac{2}{p}} .
$$

Elevating both members to $p^{\prime}=\frac{p}{p-1}$ we get

$$
\left|N^{\prime}\left(v_{n}\right)\right|^{p^{\prime}} \leq c_{1}\left|v_{n}\right|^{p}+c_{2}\left|v_{n}\right|^{2} .
$$

Then, since $v_{n}$ is bounded in $H^{1}(\Omega)$ and by (52), we have that $N^{\prime}\left(v_{n}\right)$ is bounded in $L^{p^{\prime}}$ and then it is bounded also in $\left(H^{1}(\Omega)\right)^{\prime}$. Then (51) is proved.

By the intermediate value theorem and by (51) it is easy to deduce that there exist $R$ and $M>0$ sufficiently large and $\zeta_{n} \in(0,1)$ such that

$$
\left|K_{B_{R}^{c}}\left(u+w_{n}\right)-K_{B_{R}^{c}}\left(w_{n}\right)\right| \leq\left\|K_{B_{R}^{c}}^{\prime}\left(\zeta_{n} u+\left(1-\zeta_{n}\right) w_{n}\right)\right\|_{\left(H^{1}\left(B_{R}^{c}\right)\right)^{\prime}} \cdot\|u\|_{H^{1}\left(B_{R}^{c}\right)} \leq M \cdot \varepsilon
$$

Then, by (50) and (53), we get

$$
\lim _{n \rightarrow \infty}\left|K\left(u+w_{n}\right)-K(u)-K\left(w_{n}\right)\right| \leq \varepsilon+M \cdot \varepsilon
$$

Since $\varepsilon$ is arbitrary, this limit is 0 . Then we have proved (46) . The proof of (47) is immediate.

Now we choose

$$
0<q<\frac{c}{\bar{s}} \sqrt{(m-\alpha)^{3} \alpha} .
$$

Then, by lemma 20 and (45), we have that

$$
\Lambda_{*}<\Lambda_{0} .
$$

So there exists $\mathbf{u}_{0} \in X$ and $b>0$ such that

$$
\frac{E\left(\mathbf{u}_{0}\right)}{\left|C\left(\mathbf{u}_{0}\right)\right|} \leq \Lambda_{0}-b
$$

Then we can choose $\delta>0$ such that

$$
\frac{E\left(\mathbf{u}_{0}\right)}{\left|C\left(\mathbf{u}_{0}\right)\right|}+\delta E\left(\mathbf{u}_{0}\right)^{2} \leq \Lambda_{0}-\frac{b}{2}
$$

and we define

$$
J(\mathbf{u})=\frac{E(\mathbf{u})}{|C(\mathbf{u})|}+\delta E(\mathbf{u})^{2} .
$$

Lemma 23 The functional defined by (55) is $G$-compact (where $G$ is defined by (4)). 
Proof. Let $\mathbf{u}_{n}=\left(u_{n}, \hat{u}_{n}, \theta_{n}, \Theta_{n}, \mathbf{E}_{n}, \mathbf{H}_{n}\right)$ be a minimizing sequence for $J$. Since the $G$-compactness depends on subsequences, we can take a subsequence in which all the $C\left(\mathbf{u}_{n}\right)$ have the same sign. So, to fix the ideas, we can assume that

$$
C\left(\mathbf{u}_{n}\right)>0
$$

thus we have that

$$
J\left(\mathbf{u}_{n}\right)=\frac{E\left(\mathbf{u}_{n}\right)}{C\left(\mathbf{u}_{n}\right)}+\delta E\left(\mathbf{u}_{n}\right)^{2} .
$$

It is immediate to see that $E\left(\mathbf{u}_{n}\right)=\frac{1}{2}\left\|\mathbf{u}_{n}\right\|^{2}+\int N\left(u_{n}\right) d x$ is bounded, moreover by assumption (W-iiii)

$$
\left|\int_{Q} N\left(u_{n}\right) d x\right|=o\left(\left\|\mathbf{u}_{n}\right\|_{Q}^{2}\right)
$$

We shall first show that

$$
\left\|\mathbf{u}_{n}\right\|^{2} \text { is bounded. }
$$

Now $W \geq 0$ and $E\left(\mathbf{u}_{n}\right)$ is bounded. Then, comparing (33) with (32), in order to show that $\left\|\mathbf{u}_{n}\right\|^{2}$ is bounded we have only to prove that

$$
\left\|u_{n}\right\|_{L^{2}} \text { is bounded. }
$$

Observe that, by using again the boundeness of $E\left(\mathbf{u}_{n}\right)$, we have that

$$
\int W\left(u_{n}\right) \text { and } \int\left|\nabla u_{n}\right|^{2} \text { are bounded. }
$$

By (59)) we have that

$$
\int\left|u_{n}\right|^{6} \text { is bounded. }
$$

Let $\varepsilon>0$ and set

$$
\Omega_{n}=\left\{x \in \mathbb{R}^{3}:\left|u_{n}(x)\right|>\varepsilon\right\} \text { and } \Omega_{n}^{c}=\mathbb{R}^{3} \backslash \Omega_{n} .
$$

By (59) and since $W \geq 0$, we have

$$
\int_{\Omega_{n}^{c}} W\left(u_{n}\right) \text { is bounded . }
$$

By (W-ii) we can write

$$
W(s)=\frac{m}{2} s^{2}+o\left(s^{2}\right) .
$$

Then, if $\varepsilon$ is small enough, there is a constant $c>0$ such that

$$
\int_{\Omega_{n}^{c}} W\left(u_{n}\right) \geq c \int_{\Omega_{n}^{c}} u_{n}^{2} .
$$


By (61) and (62) we get that

$$
\int_{\Omega_{n}^{c}} u_{n}^{2} \text { is bounded. }
$$

On the other hand

$$
\int_{\Omega_{n}} u_{n}^{2} \leq\left(\int_{\Omega_{n}}\left|u_{n}\right|^{6}\right)^{\frac{1}{3}} \cdot \operatorname{meas}\left(\Omega_{n}\right)^{\frac{2}{3}} .
$$

By (60) we have that

$$
\text { meas }\left(\Omega_{n}\right) \text { is bounded. }
$$

By (64), (65), (60) we get that

$$
\int_{\Omega_{n}} u_{n}^{2} \text { is bounded. }
$$

So (58) follows from (63) and (66).

We shall now prove that there exists a subsequence $u_{n^{\prime}}$ of $u_{n}$ and

$$
\left\{j_{n^{\prime}}\right\} \subset \mathbb{I}=\left\{j \in \mathbb{Z}^{3}: C_{Q_{j}}\left(u_{n}\right)>0\right\}
$$

such that

$$
g_{j_{n^{\prime}}} u_{n^{\prime}} \rightarrow \bar{u} \neq 0 \text { weakly in } H^{1}\left(\mathbb{R}^{3}\right) .
$$

To this end we show first that for any $n$ sufficiently large there is $j_{n} \in \mathbb{I}$ such that (see (54))

$$
\frac{E_{Q_{j_{n}}}\left(\mathbf{u}_{n}\right)}{C_{Q_{j_{n}}}\left(\mathbf{u}_{n}\right)} \leq \Lambda_{0}-\frac{b}{2} .
$$

Since $\mathbf{u}_{n}$ is a minimizing sequence for $J$, by (54) there is $M>0$ such that, for any $n \geq M$ we have

$$
\begin{aligned}
\Lambda_{0}-\frac{b}{2} & \geq J\left(\mathbf{u}_{n}\right)=\frac{E\left(\mathbf{u}_{n}\right)}{C\left(\mathbf{u}_{n}\right)}+\delta E\left(\mathbf{u}_{n}\right)^{2} \\
& \geq \frac{E\left(\mathbf{u}_{n}\right)}{C\left(\mathbf{u}_{n}\right)}=\frac{\sum_{j} E_{Q_{j}}\left(\mathbf{u}_{n}\right)}{\sum_{j} C_{Q_{j}}\left(\mathbf{u}_{n}\right)} \\
& \geq \frac{\sum_{j \in \mathbb{I}} E_{Q_{j}}\left(\mathbf{u}_{n}\right)}{\sum_{j \in \mathbb{I}} C_{Q_{j}}\left(\mathbf{u}_{n}\right)} .
\end{aligned}
$$

Now arguing by contradiction assume that (69) does not hold, namely assume that

$$
\text { for any } j \in \mathbb{I} \text { : } \frac{E_{Q_{j}}\left(\mathbf{u}_{n}\right)}{C_{Q_{j}}\left(\mathbf{u}_{n}\right)}>\Lambda_{0}-\frac{b}{2} \text {. }
$$


Then by (70) and (71) we have

$$
\Lambda_{0}-\frac{b}{2} \geq \frac{\sum_{j \in \mathbb{I}} E_{Q_{j}}\left(\mathbf{u}_{n}\right)}{\sum_{j \in \mathbb{I}} C_{Q_{j}}\left(\mathbf{u}_{n}\right)}>\frac{\sum_{j \in \mathbb{I}} C_{Q_{j}}\left(\mathbf{u}_{n}\right)\left(\Lambda_{0}-\frac{b}{2}\right)}{\sum_{j \in \mathbb{I}} C_{Q_{j}}\left(\mathbf{u}_{n}\right)}=\Lambda_{0}-\frac{b}{2} .
$$

So we get a contradiction and then (69) is proved.

Clearly $g_{j_{n}} \mathbf{u}_{n}$ is bounded, then there is a subsequence $g_{j_{n^{\prime}}} \mathbf{u}_{n^{\prime}}$ such that

$$
g_{j_{n^{\prime}}} u_{n^{\prime}} \rightarrow \bar{u} \text { weakly in } H^{1}\left(\mathbb{R}^{3}\right), g_{j_{n^{\prime}}} \theta_{n^{\prime}} \rightarrow \bar{\theta} \text { weakly in } L^{2}\left(\mathbb{R}^{3}\right) \text {. }
$$

Here clearly we have set $\left(g_{j_{n^{\prime}}} u_{n^{\prime}}\right)(x)=u_{n^{\prime}}\left(x-j_{n^{\prime}}\right)$ and $\left(g_{j_{n^{\prime}}} \theta_{n^{\prime}}\right)(x)=\theta_{n^{\prime}}(x-$ $j_{n^{\prime}}$.

We show that

$$
\left\|g_{j_{n^{\prime}}} \mathbf{u}_{n^{\prime}}\right\|_{Q} \text { does not converge to } 0 \text {. }
$$

Arguing by contradiction assume that

$$
\left\|g_{j_{n^{\prime}}} \mathbf{u}_{n^{\prime}}\right\|_{Q} \rightarrow 0
$$

Then by (69) and (56) we have

$$
\Lambda_{0}-\frac{b}{2} \geq \frac{E_{Q_{j_{n^{\prime}}}}\left(\mathbf{u}_{n^{\prime}}\right)}{C_{Q_{j_{n^{\prime}}}}\left(\mathbf{u}_{n^{\prime}}\right)} \geq \frac{\frac{1}{2}\left\|g_{j_{n^{\prime}}} u_{n^{\prime}}\right\|_{Q}^{2}+o\left(\left\|g_{j_{n^{\prime}}} u_{n^{\prime}}\right\|_{Q}^{2}\right)}{C_{Q}\left(g_{j_{n^{\prime}}} \mathbf{u}_{n^{\prime}}\right)} .
$$

So by (75), by definition of $\Lambda_{0}$ and passing to the limit in (76), we get

$$
\Lambda_{0}-\frac{b}{2} \geq \Lambda_{0}
$$

which gives the contradiction and (74) holds.

By (74) and (73) we deduce that

$$
\bar{u} \neq 0 \text { in } Q .
$$

In fact, arguing by contradiction, assume that $\bar{u}=0$ in $Q$. By (73) we get $g_{j_{n^{\prime}}} u_{n^{\prime}} \rightarrow \bar{u}=0$ strongly in $L^{2}(Q)$ and $g_{j_{n^{\prime}}} \theta_{n^{\prime}} \rightarrow \bar{\theta}$ weakly in $L^{2}(Q)$, then

$$
C_{Q}\left(g_{j_{n^{\prime}}}, \mathbf{u}_{n^{\prime}}\right)=\int_{Q} g_{j_{n^{\prime}}} u_{n^{\prime}} g_{j_{n^{\prime}}} \theta_{n^{\prime}} \rightarrow 0
$$

By (76) and (77) we deduce that

$$
E_{Q}\left(g_{j_{n^{\prime}}} \mathbf{u}_{n^{\prime}}\right) \rightarrow 0
$$

and consequently $\left\|g_{j_{n^{\prime}}} \mathbf{u}_{n^{\prime}}\right\|_{Q} \rightarrow 0$ contradicting (74). So we conclude that $\bar{u} \neq 0$ in $Q$.

From now on we write for simplicity $g_{j_{n}} \mathbf{u}_{n}$ instead of $g_{j_{n^{\prime}}} \mathbf{u}_{n^{\prime}}$ and set 


$$
g_{j_{n}} \mathbf{u}_{n}=\overline{\mathbf{u}}+\mathbf{w}_{\mathbf{n}} .
$$

with $\mathbf{w}_{\mathbf{n}} \rightarrow 0$ weakly.

We finally show that there is no splitting, namely that $\mathbf{w}_{\mathbf{n}} \rightarrow \mathbf{0}$ strongly.

By the $G$-invariance of $E$ and $C$ and lemma 22, we have

$$
\begin{aligned}
J_{*} & :=\lim J\left(g_{j_{n}} \mathbf{u}_{n}\right)=\lim \frac{E\left(g_{j_{n}} \mathbf{u}_{n}\right)}{C\left(g_{j_{n}} \mathbf{u}_{n}\right)}+\delta E\left(g_{j_{n}} \mathbf{u}_{n}\right)^{2} \\
& =\lim \frac{E(\overline{\mathbf{u}})+E\left(\mathbf{w}_{n}\right)+o(1)}{C(\overline{\mathbf{u}})+C\left(\mathbf{w}_{n}\right)+o(1)}+\delta\left[E(\overline{\mathbf{u}})+E\left(\mathbf{w}_{n}\right)+o(1)\right]^{2} \\
& =\lim \frac{E(\overline{\mathbf{u}})+E\left(\mathbf{w}_{n}\right)}{C(\overline{\mathbf{u}})+C\left(\mathbf{w}_{n}\right)}+\delta E(\overline{\mathbf{u}})^{2}+\delta E\left(\mathbf{w}_{n}\right)^{2}+2 \delta E(\overline{\mathbf{u}}) E\left(\mathbf{w}_{n}\right) \\
& \geq \lim \frac{E(\overline{\mathbf{u}})+E\left(\mathbf{w}_{n}\right)}{|C(\overline{\mathbf{u}})|+\left|C\left(\mathbf{w}_{n}\right)\right|}+\delta E(\overline{\mathbf{u}})^{2}+\delta E\left(\mathbf{w}_{n}\right)^{2}+2 \delta E(\overline{\mathbf{u}}) E\left(\mathbf{w}_{n}\right) \\
& \geq \lim \left[\min \left(\frac{E(\overline{\mathbf{u}})}{|C(\overline{\mathbf{u}})|}, \frac{E\left(\mathbf{w}_{n}\right)}{\left|C\left(\mathbf{w}_{n}\right)\right|}\right)\right]+\delta E(\overline{\mathbf{u}})^{2}+\delta E\left(\mathbf{w}_{n}\right)^{2}+2 \delta E(\overline{\mathbf{u}}) E\left(\mathbf{w}_{n}\right) \\
& \geq \lim \left[\min \left(J(\overline{\mathbf{u}}), J\left(\mathbf{w}_{n}\right)\right)\right]+2 \delta E(\overline{\mathbf{u}}) E\left(\mathbf{w}_{n}\right) \geq\left(\operatorname{since} J_{*}=\inf J\right) \\
& \geq \lim \left[J_{*}+2 \delta E(\overline{\mathbf{u}}) E\left(\mathbf{w}_{n}\right)\right]=J_{*}+2 \delta E(\overline{\mathbf{u}}) \lim E\left(\mathbf{w}_{n}\right) .
\end{aligned}
$$

Then

$$
2 \delta E(\overline{\mathbf{u}}) \lim E\left(\mathbf{w}_{n}\right) \leq 0
$$

and since $E(\overline{\mathbf{u}}) \neq 0$, we have that

$$
\lim E\left(\mathbf{w}_{n}\right)=0
$$

and hence $\mathbf{u}_{n} \rightarrow \overline{\mathbf{u}}$ strongly. Then $J$ is $G$-compact.

Proof of Th. 18, We shall use Theorem 15, Obviously assumptions (EC-1) and (EC-2) are satisfied with $G$ given by (4). Then by lemma 23 and Th. 15 we have the existence of soliton solutions. In order to prove that they form a family dependent of $\delta$, it is sufficient to prove that $\delta_{1} \neq \delta_{2}$ in the definition (55) of $J$ implies $\mathbf{u}_{\delta_{1}} \neq g \mathbf{u}_{\delta_{2}}$ for every $g \in G$. We argue indirectly and assume that $\mathbf{u}_{\delta_{1}}=g \mathbf{u}_{\delta_{2}}$ for some $g \in G$. Then

$$
\frac{E\left(g \mathbf{u}_{\delta_{2}}\right)}{\left|C\left(g \mathbf{u}_{\delta_{2}}\right)\right|}+\delta_{2} E\left(g \mathbf{u}_{\delta_{2}}\right)^{2}=\frac{E\left(\mathbf{u}_{\delta_{1}}\right)}{\left|C\left(\mathbf{u}_{\delta_{1}}\right)\right|}+\delta_{1} E\left(\mathbf{u}_{\delta 1}\right)^{2}
$$

and so, since $g \mathbf{u}_{\delta_{2}}=\mathbf{u}_{\delta_{1}}$,

$$
\begin{aligned}
0 & =\frac{E\left(g \mathbf{u}_{\delta_{2}}\right)}{\left|C\left(g \mathbf{u}_{\delta_{2}}\right)\right|}+\delta_{2} E\left(g \mathbf{u}_{\delta_{2}}\right)^{2}-\left(\frac{E\left(\mathbf{u}_{\delta_{1}}\right)}{\left|C\left(\mathbf{u}_{\delta_{1}}\right)\right|}+\delta_{1} E\left(\mathbf{u}_{\delta 1}\right)^{2}\right) \\
& =\left(\delta_{2}-\delta_{1}\right) E\left(\mathbf{u}_{\delta_{1}}\right)^{2}
\end{aligned}
$$

Then, since $\delta_{1} \neq \delta_{2}, E\left(\mathbf{u}_{\delta_{1}}\right)=0$ and so $\mathbf{u}_{\delta_{1}}=0$ which is a contradiction. 
Proof of Th. 19, Let $\mathbf{u}_{\delta}=\left(u_{\delta}, 0, \theta_{\delta}, 0, \mathbf{E}_{\delta}, \mathbf{0}\right)$ be a minimizer of $J$ (defined by (55)) on $X=\{\mathbf{u} \in \mathcal{H}: \nabla \cdot \mathbf{E}=-q \theta u, \nabla \cdot \mathbf{H}=0\}$. By Theorem 12 any $\mathbf{u} \in$ $X$, with energy $E\left(\mathbf{u}_{\delta}\right)$ and charge $C\left(\mathbf{u}_{\delta}\right)$, is a soliton. So, in particular also $\mathbf{u}_{\delta}$ is a soliton. Clearly $\mathbf{u}_{\delta}$ minimizes also the energy $E$ (see (33)) on the manifold

$$
X_{\delta}=\left\{\mathbf{u} \in X: C(\mathbf{u})=C\left(\mathbf{u}_{\delta}\right)=\sigma_{\delta}\right\} .
$$

If we write $\mathbf{E}=-\nabla \phi$, the constraint $\nabla \cdot \mathbf{E}=-q \theta u$ becomes

$$
\Delta \phi=q \theta u .
$$

So $\mathbf{u}_{\delta}$ is a critical point of $E$ on the manifold (in $\mathcal{H}$ ) made up by those $\mathbf{u}=$ $(u, 0, \theta, 0, \nabla \phi, \mathbf{0})$ satisfying the constraints

$$
\begin{gathered}
\Delta \phi=q \theta u \\
C(\mathbf{u})=\int \theta u d x=\sigma_{\delta} .
\end{gathered}
$$

Therefore, for suitable Lagrange multipliers $\lambda \in \mathbb{R}, \xi \in \mathcal{D}^{1,2}\left(\mathcal{D}^{1,2}\right.$ is the closure of $C_{0}^{\infty}$ with respect to the norm $\|\nabla \phi\|_{L^{2}}$, , we have that $\mathbf{u}_{\delta}$ is a critical point of

$$
E_{\lambda, \xi}(\mathbf{u})=E(\mathbf{u})+\lambda\left(\int \theta u d x-\sigma_{\delta}\right)+\langle\xi,-\Delta \phi+q \theta u\rangle
$$

where $\langle\cdot, \cdot\rangle$ denotes the duality map in $\mathcal{D}^{1,2}$. It is easy to show that $E_{\lambda, \xi}^{\prime}\left(\mathbf{u}_{\delta}\right)=$ 0 gives the equations

$$
\begin{aligned}
-\Delta u_{\delta}+W^{\prime}\left(u_{\delta}\right)+\lambda \theta_{\delta}+q \xi \theta_{\delta} & =0 \\
-\Delta \phi_{\delta} & =\Delta \xi \\
\theta_{\delta}+\lambda u_{\delta}+q \xi u_{\delta} & =0 .
\end{aligned}
$$

From (83) we get $\xi=-\phi_{\delta}$, so (82) and (84) become

$$
\begin{aligned}
-\Delta u_{\delta}+W^{\prime}\left(u_{\delta}\right)+\theta_{\delta}\left(\lambda-q \phi_{\delta}\right) & =0 \\
\left(\lambda-q \phi_{\delta}\right) u_{\delta} & =-\theta_{\delta} .
\end{aligned}
$$

From the above equations we clearly get (36). (37) is given by the constraint (79). 


\section{References}

[1] Badiale M., Benci V., Rolando S., Solitary waves: physical aspects and mathematical results, Rend. Sem. Math. Univ. Pol. Torino 62 (2004), 107-154.

[2] Bellazzini J.,Benci V.,Bonanno C., Micheletti A.M., Solitons for the Nonlinear Klein-Gordon-Equation, (arXiv:0712.1103), to apper on Advanced of Nonlinear Studies (2010).

[3] Bellazzini J., Benci V., Bonanno C., Sinibaldi E., Hylomorphic solitons in the nonlinear Klein-Gordon equation, Dynamics of Partial Differential Equations, 6 (2009), 311-336.

[4] Benci V, Hylomorphic solitons, Milan J. Math. 77 (2009), 271-332.

[5] Benci V. Fortunato D., Solitary waves in Abelian Gauge Theories, Adv. Nonlinear Stud. 3 (2008), 327-352.

[6] Benci V. Fortunato D., Existence of hylomorphic solitary waves in Klein-Gordon and in Klein-Gordon-Maxwell equations, Rend. Lincei Mat. Appl. supplemento, 20 (2009), 243-279.

[7] Benci V. Fortunato D., Solitary waves in the Nonlinear Wave equation and in Gauge Theories, Journal of fixed point theory and Applications, 1, n.1 (2007), 61-86.

[8] Benci V. Fortunato D., Spinning Q-balls for the Klein-GordonMaxwell Equations, Commun. Math. Phys., 295 (2010), 639-668. doi: $10.1007 / \mathrm{s} 00220-010-0985-\mathrm{Z}$

[9] Benci V. Fortunato D., Hylomorphic solitons on lattices, Discrete and Continuous Dynamical Systems, 28 (2010), 875-897 doi:10.3934/dcds.2010.28.875.

[10] Benci V. Fortunato D., Hamiltonian formulation of the Klein-GordomMaxwell equations, in preparation.

[11] Bonanno C., Existence and multiplicity of stable bound states for the nonlinear Klein-Gordon equation. Nonlinear Analysis 72 (2010), 20-31.

[12] Berestycki H., Lions P.L., Nonlinear scalar field equations, I - Existence of a ground state, Arch. Rational Mech. Anal. 82 (1983), 313-345.

[13] Coleman S., Q-Balls, Nucl. Phys. B262 (1985) 263-283; erratum: B269 (1986) 744-745.

[14] Gelfand I.M., Fomin S.V., Calculus of Variations, Prentice-Hall, Englewood Cliffs, N.J. 1963. 
[15] Klainerman, S., Machedon, M., On the Maxwell-Klein-Gordon equation with finite energy, Duke Math. J. 74 (1994), 19-44.

[16] Mugnai D., Solitary waves in Abelian Gauge Theories with strongly nonlinear potentials, Ann. Inst. H. Poincarè, to appear.

[17] Rajaraman R., Solitons and instantons, North-Holland, Amsterdam 1989.

[18] Rosen G., Particlelike solutions to nonlinear complex scalar field theories with positive-definite energy densities, J. Math. Phys. 9 (1968), 996-998.

[19] Shatah J., Stable Standing waves of Nonlinear Klein-Gordon Equations, Comm. Math. Phys., 91, (1983), 313-327.

[20] Vilenkin A., Shellard E.P.S., Cosmic strings and other topological defects, Cambridge monographs on mathematical physics, 1994.

[21] Yang Y., Solitons in Field Theory and Nonlinear Analysis, Springer, New York, Berlin, 2000. 